\title{
A Method for Estimating Chirp Rate of a Linear Frequency Modulated Signal
}

\author{
A. Serbes
}

\begin{abstract}
This paper proposes a fast and accurate chirp-rate estimation algorithm in noisy environment. The proposed method searches the inverse maximum magnitude of the angle-sweep fractional Fourier domain and finds the optimum angle, which provides an estimate for the chirp-rate. In order to accelerate the search process, we propose to use the Fibonacci search algorithm, which provides a sub-optimal search scheme in terms of computational complexity. Simulation results show the efficiency of the proposed algorithm.
\end{abstract}

Index Terms-Fractional Fourier transform, chirp-rate, linear frequency modulated signals, estimation, Cramer-Rao lower bound.

\section{INTRODUCTION}

$\mathrm{L}$ INEAR frequency modulated (LFM) signals are employed in a relatively diverse areas, such as radar [1], sonar [2], automotive [3], aerospace [4], ultrasound [5], and communications [6]. One of the most important problems when dealing with LFM signals is to estimate one, or all of its parameters. An LFM signal in a noisy environment can be modeled as

$$
c(t)=A \exp \left[j \pi\left(m t^{2}+2 f_{0} t\right)\right]+w(t),
$$

where $m$ is the chirp rate, $f_{0}$ is the frequency-shift, and $A$ is the complex amplitude. In this problem, the noise $w(t)$ is assumed to be a complex circular additive white Gaussian with variance $\sigma_{w}^{2}$.

Radar return signals of a moving object can be modeled by an LFM signal. Therefore, estimating chirp rates of the radar signals provide an estimate of the relative velocity of the target. Moreover, LFMCW radars emit LFM-type signals in order to estimate both velocity and the distance at the same time. Therefore, estimating the chirp-rate of an LFM signal play a critical role in many applications. The asymptotic Cramer-Rao lower bound for estimating the chirp rate of a single LFM signal can be expressed by

$$
\operatorname{var}(\widehat{m})=\frac{90}{\pi^{2} N^{5} T_{s}^{4} \gamma}
$$

where $\widehat{m}$ is the estimated chirp rate and $\gamma$ is the signal-to-noise ratio (SNR).

A. SERBES, is with Department of Electronics and Communications Engineering of Yildiz Technical University, Istanbul, Turkey, (e-mail: aserbes@yildiz.edu.tr) (iD

Manuscript received August 16, 2017; accepted Dec 23, 2017. DOI: 10.17694/bajece.369239
Here, the LFM signal sampled with a sampling period $T_{s}$ and $N$ samples are taken. The maximum likelihood estimators [7-8] for estimating parameters of the LFM signal requires a multidimensional parameter search algorithm in the search space. Therefore it is computationally inefficient. Wigner distributionbased methods [9-10] are also of high performance; however they also lack computational ease. Maximum amplitude method [11] can also be used to estimate the chirp rate of a signal.

In this paper, we present a novel, fast and accurate chirp-rate estimation algorithm based on the fractional Fourier transform (FrFT) and the Fibonacci search. The FrFT is a very-well known transform and has found itself many application areas, when the frequency of the signal of interest varies linearly in time. The FrFT is a natural domain for LFM signals, since the kernel of the FrFT itself is constituted of LFM signals. Throughout the FrFT domains, there exists a special domain, in which the energy of the LFM signals are most concentrated [1113]. Using the energy preservation property of the FrFT, which states that the signal's amplitude increases when the energy concentrates, we propose a novel method for estimating the optimum transformation angle of an LFM signal. Since the optimum transformation angle is related to the chirp rate, the problem of estimating the chirp rate is then reduced to a singledimensional search in the FrFT domains. In order to accelerate the search process, we propose to use the celebrated Fibonacci search [14], which requires far less computations. Simulation results show that the proposed method performs very well with less computational requirements.

The rest of the paper is organized as follows, Section II introduces the FrFT briefly and concludes with how the maximum magnitude method can be used for chirp rate estimation. Section III discloses the proposed method for estimating the chirp-rate and the Fibonacci search algorithm that accelerates the search process. Computer simulations are given in Section IV. The paper concludes in Section V.

\section{PRELIMINARIES}

In this section, we first give a preliminary definition of the FrFT and the section is concluded after the discussion relationship between the chirp rate of an LFM signal and the FrFT.

\section{A. The Fractional Fourier Transform}

The FrFT [15] is a linear and unitary transform. If $f(u)$ is a square integrable function, then the FrFT of this function is defined by 


$$
\begin{gathered}
\mathcal{F}^{\alpha}\{f\}(u)=\sqrt{1-j \cot (\alpha)} \int_{-\infty}^{\infty} f(\mu) \exp \left[j \pi \left(\cot (\alpha) \mu^{2}\right.\right. \\
\left.\left.-2 \csc (\alpha) u \mu+\cot (\alpha) u^{2}\right)\right] d \mu,
\end{gathered}
$$

where $\alpha$ is the transformation angle. The FrFT becomes the conventional FT for $\alpha=\pi / 2$ and inverse FT for $\alpha=-\pi / 2$. Therefore, FT is only a special case of the FrFT and for the other values of angles; the FrFT can be considered as an interpolation between the original signal, and the FT. For example, if $\alpha=2 \pi$ and $\alpha=\pi$, the transform appears to be the identity transform and the counter-identity transform, respectively. The FrFT is an energy-preserving transform as implied by the unitarity. Two consecutive FrFTs with angles $\alpha_{1}$ and $\alpha_{2}$ yield a single FrFT with the angle $\alpha_{1}+\alpha_{2}$, where the FrFT transformation angle $\alpha$ is periodic with $2 \pi$.

One of the most interesting properties of the FrFT is that it rotates the transformed signal in the time-frequency domain, i.e., the Wigner distribution of the resultant signal is rotated with the transform angle $\alpha$, in the clockwise direction. In other words, the FrFT rotates the time frequency axis in the counterclockwise direction. This property is illustrated in Fig. 1.

From (), it can be seen that the basis functions of the FrFT are the chirp functions. Therefore, the FrFT is the natural domain for LFM-type signals. In the optimum angle, the FrFT converts an LFM signal into a Dirac-delta distributed signal, where it is well-known [12-13] that the optimum angle $\alpha_{\text {opt }}$ is

$$
\alpha_{\text {opt }}=\arctan (m)+\frac{\pi}{2}+k \pi,
$$

where $m$ is the actual chirp rate of the LFM signal of interest and $k$ is any integer. Since the transform angle is a continuous function of the transform, it generally requires a search in the FrFT domain to find out the optimum transformation angle, which is generally computationally demanding.

\section{B. Chirp Rate Estimation in the Fractional Fourier Domains}

It is a very well-known fact that the FrFT can be used as a powerful tool for estimating the chirp rate of an LFM signal. The most widely used algorithm for estimating the chirp rate is the maximum magnitude method. This method searches for the angle that produces the maximum magnitude throughout all the angles between $[0, \pi]$ expressed by

$$
\hat{\alpha}_{\text {opt }}=\underset{\alpha}{\operatorname{argmax}} P(\alpha),
$$

where $P(\alpha)$ is the maximum of the magnitude square FrFT at the angle $\alpha$, given by,

$$
P(\alpha)=\max \left\{\left|\mathcal{F}^{\alpha}\{c\}(u)\right|^{2}\right\} \text {. }
$$

The expected value of the resultant estimate $\hat{\alpha}$ is known to give the optimum transform angle, $\alpha_{\text {opt }}$. Therefore, an estimate of the chirp rate can be found by

$$
\widehat{m}=\tan \left(\hat{\alpha}_{o p t}-\frac{\pi}{2}\right) .
$$

As the maximum magnitude method searches through the angles, the LFM signal gets more and more compact when the search angle gets closer to the optimum angle. As a result of the preservation of the energy, the magnitude increases in order to make the total energy constant in the transform domain. The magnitude attains its maximum at the optimum transform domain, which converts the signal into an impulse, making the maximum magnitude method an optimum chirp-rate estimation method.

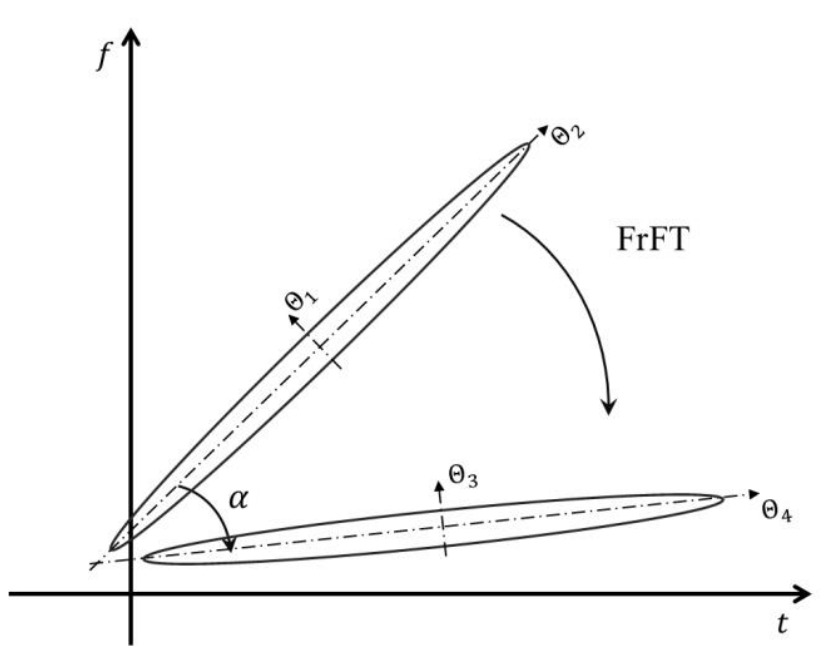

Fig. 1. Illustration of the rotation property of the FrFT in the timefrequency domain.

The only limiting factor of the usage of FrFT-based methods for estimating the chirp rate is its relatively high computational cost of digital computation of the FrFT. In the FrFT-domain, only a one-dimensional search is required to estimate the chirprate by using the maximum magnitude method. However, digital calculation of a single FrFT requires $\mathcal{O}(N \log N)$ complex multiplications and additions. Therefore, fast angle search algorithms are required. In the next section we propose a method that employs the FrFT to estimate the chirp-rate of an LFM signal based on.

\section{A. Minimum Inverse Magnitude}

\section{PRoposed Method}

We propose that, instead of using the maximum magnitude method, minimum inverse magnitude method can also be used as a substitute. In the minimum inverse magnitude, an estimate of the optimum angle is found by,

$$
\hat{\alpha}_{\text {opt }}=\min _{\alpha} J(\alpha),
$$

where,

$$
J(\alpha)=\frac{1}{P(\alpha)}
$$

which simply finds the minimum of the inverse of the maximum magnitude. However, calculating each point in the search space requires $\mathcal{O}(N \log N)$ complex multiplications and additions to calculate the FrFT. Therefore, instead of employing a brute-search algorithm, we propose to use the Fibonacci search.

\section{B. Fibonacci Search}

Fibonacci search is one of the well-known near-optimum search methods. In this method, a local search for the minimum of a cost function is evaluated cost-effective. The Fibonacci search algorithm finds a minimum of a cost function $f(x)$ in the interval of $[a, b]$.

In the Fibonacci algorithm, we select two points $x_{1}$ and $x_{2}$ in the range $[a, b]$ and evaluate the function at these two points 
$f\left(x_{1}\right)$ and $f\left(x_{2}\right)$, where $x_{1}<x_{2}$ and $\left(x_{1}-a\right)=\left(b-x_{2}\right)$. Here, we assume that $f(a)$ and $f(b)$ are already known. If $f\left(x_{1}\right)<f\left(x_{2}\right)$, then we confine the new search interval to $\left[a, x_{2}\right]$. Otherwise, the new search interval is $\left[x_{1}, b\right]$. After updating the new interval, only a new point $x_{3}$ is selected inside the new interval and the other two points can be used. For example, if the new interval is $\left[a, x_{2}\right]$, the new point is selected such that $a<x_{3}<x_{2}$. Then, $f\left(x_{3}\right)$ is evaluated. If $f\left(x_{3}\right)<$ $f\left(x_{1}\right)$, then we confine the new interval as $\left[a, x_{1}\right]$. Otherwise, the new search interval is set to $\left[x_{3}, x_{2}\right]$. The beauty of the Fibonacci search method comes from the way of selecting internal points. The search points are selected such that only a single evaluation of the cost function is required at each iteration, except for the first iteration, which requires two evaluations. The algorithm stops when the search interval is smaller than a tolerance value $\epsilon$. The proposed Fibonacci search algorithm for finding the minimum of inverse maximum magnitude is summarized in Algorithm 1, where $F_{k}=F_{k-1}+$ $F_{k-2}$ is the $k$-th Fibonacci number of the order $k>1$.

In our realizations, we choose $a=0$ and $b=2 \pi$, so that the whole FrFT domain is our search domain. The number of required iterations is determined by the tolerance value $\epsilon$. It is a well-kown fact that a Fibonacci number can be approximated by $F_{k} \approx \frac{\sqrt{5}}{2} r^{k+1}$ for $k \geq 3$, where $r \approx 1.618$ is the golden ratio. Therefore, the required number of FrFTs can be found as,

Algorithm 1: The employed Fibonacci search method that finds an estimate of the chirp-rate, where the search is performed in the FrFT angles between 0 and $2 \pi$.

$$
\begin{aligned}
& n \leftarrow \underset{n}{\operatorname{argmin}}\left\{F_{n} \leq \frac{(b-a)}{\epsilon}\right\} \\
& \alpha_{1} \leftarrow a+\frac{F_{n-2}}{F_{n}}(b-a) \\
& \alpha_{2} \leftarrow a+\frac{F_{n-1}}{F_{n}}(b-a) \\
& \text { while } k<n-1 \\
& \text { if } J\left(\alpha_{1}\right)>J\left(\alpha_{2}\right) \\
& \quad a \leftarrow \alpha_{1} ; \\
& \quad \alpha_{1} \leftarrow \alpha_{2} ; \\
& \quad \alpha_{2} \leftarrow a+\frac{F_{n-k-1}}{F_{n-k}}(b-a) ; \\
& \text { else } \\
& \quad b \leftarrow \alpha_{2} ; \\
& \quad \alpha_{2} \leftarrow \alpha_{1} ; \\
& \quad \alpha_{1} \leftarrow a+\frac{F_{n-k-2}}{F_{n-k}}(b-a) ; \\
& \text { end } \\
& k \leftarrow k+1 ; \\
& \text { end } \quad k \\
& \hat{\alpha_{o p t}} \leftarrow \frac{\alpha_{1}+\alpha_{2}}{2} ; \\
& \hat{m}=\tan \left(\hat{\alpha}_{o p t}-\frac{\pi}{2}\right)
\end{aligned}
$$

$$
N_{\text {FrFT }}=\left\lceil\log _{r}\left(\frac{2}{\sqrt{5}} \frac{b-a}{\epsilon}\right)-2\right\rceil,
$$

where $\lceil\cdot]$ denotes the ceil operator. For a setting of $\epsilon=10^{-5}$, $b=\pi$, and $a=0$, the number of required FrFTs is only 26. As the computational complexity of digital computation of the FrFT is of $\mathcal{O}(N \log N)$ [16], our proposed algorithm is of $\mathcal{O}\left(N_{F r F T} N \log N\right)$ computational cost.

The next section discusses the estimation performance of the proposed chirp-rate estimator.

\section{Simulations AND RESUlts}

In order to evaluate the performance of the proposed method, we generate a discrete-chirp signal,

$$
\begin{gathered}
c[n]=A \exp \left\{j \pi\left[m \times\left(n T_{s}\right)^{2}+2 f_{0}\left(n T_{s}\right)\right]\right\}+w[n], \\
n=0,1 \cdots, N-1,
\end{gathered}
$$

where we take the sampling rate $T_{s}=1 / \sqrt{N}$, chirp rate $m=$ 0.2 , frequency shift $f_{0}=0.1$, and amplitude $A=1$, and the number of the samples $N=1024 . w[n]$ is the additive white Gaussian noise with variance $\sigma_{w}^{2}$.

In the noiseless case, the cost function $J(\alpha)$ for the LFM signal is plotted in Figure 2. It is clear that the cost function is an almost unimodal function attaining its minimum at the optimum angle $\alpha_{\text {opt }}=\arctan (m)+\pi / 2$.

In the noisy case, estimation performance is compared to the CRLB in Figure 3. Performance criteria is taken as the MSE of the chirp-rate estimation, given by

$$
M S E=\frac{1}{M} \sum_{k=1}^{M}(\widehat{m}-m),
$$

where $\hat{m}$ is the estimated chirp rate and $m$ is the actual chirp rate of the signal. $M$ is the number of Monte-Carlo simulations, which we take as $10^{4}$.

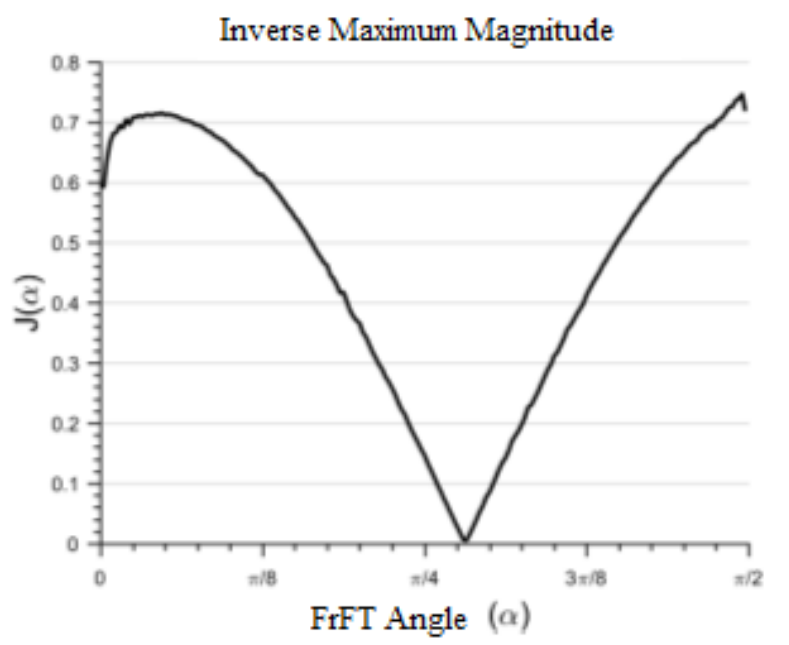

Figure 2. Inverse maximum magnitude cost function of a chirp signal.

Estimation performance of a the LFM chirp rate is plotted in Figure 3, which shows that MSE gets closer to the CRLB after a specific value of SNR. For the SNR values below the 
threshold, estimation performance is highly degraded, which is also known as the maximum likelihood threshold. For this simulation we choose the Fibonacci parameter as $\epsilon=\frac{10^{-5}}{\pi}$. For this value of $\epsilon$, only 26 iterations are required to estimate the chirp-rate. Therefore, the computational cost of our proposed estimator is only in the order of $\mathcal{O}(26 N \log N)$.

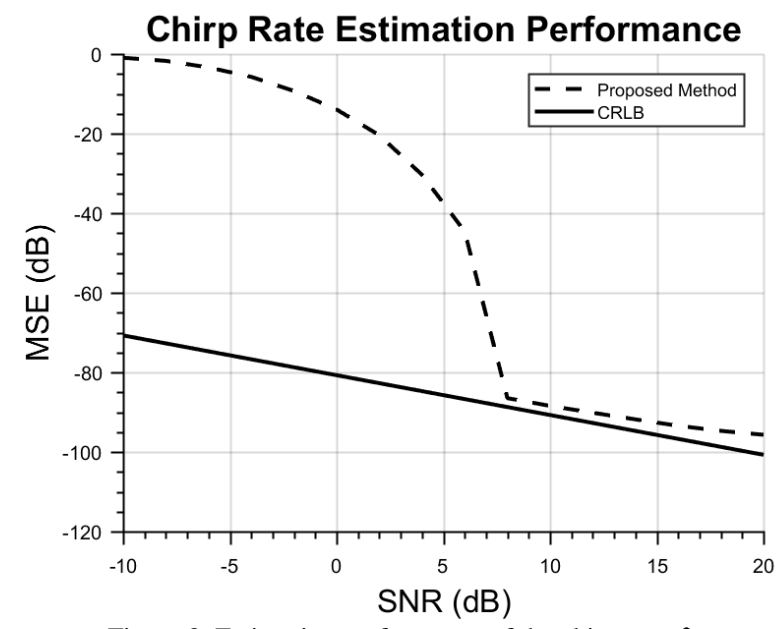

Figure 3. Estimation performance of the chirp rate $\widehat{m}$.

Figure 3 also shows that, for SNR values greater than $7 \mathrm{~dB}$, MSE of the estimation are very low. For most of the applications, this kind of estimation accuracy is highly satisfactory.

\section{CONCLUSIONS}

In this work, we have presented a fast and accurate way to estimate the chirp-rate of an LFM signal. The problem of estimating the chirp-rate is reduced to finding the minimum of a cost function, $J(\alpha)$. Taking the advantage of the relationship between the index that minimizes $J(\alpha)$ and the chirp rate, chirprate of the LFM signal is estimated. Instead of a brute-search, we propose to use the celebrated Fibonacci search in order to accelerate the search process. Simulation results show that the proposed method produces highly accurate estimation results.

\section{REFERENCES}

[1] A.G. Stove, "Linear FMCW radar techniques," Proc. Inst. Elect. Eng., pt. F, pp. 343-350, Oct. 1992.

[2] P.R. Atkins, T. Collins, K.G. Foote, "Transmit-signal design and processing strategies for sonar target phase measurement," IEEE J. Sel. Topics Signal Process., vol. 1, no. 1, Jun. 2007.

[3] S. Patole, M. Torlak, D. Wang, and M. Ali, "Automotive radars: A review of signal processing techniques," IEEE Signal Proc. Mag., col. 34, no. 2, 2017.

[4] J. Muoz-Ferraras, F. Perez-Martinez, M. Burgos-Garcia, "Helicopter classification with a high resolution LFMCW radar," IEEE Trans. Aerosp. Electron. Syst., vol. 45, no. 4, pp. 1373-1384, Jan. 2010.

[5] T. Misaridis, J. A. Jensen, "Use of modulated excitation signals in medical ultrasound. Part I: basic concepts and expected benefits," IEEE Trans. Ultrason., Ferroelect., Freq. Control, vol. 52, no. 2, pp. 177-191, Feb. 2005

[6] M. Martone, "A multicarrier system based on the fractional Fourier transform for time-frequency-selective channels," IEEE Trans. Commun., vol. 49, no. 6, pp. 1011-1020, Jun. 2001.
[7] P.M. Djuric and S. M. Kay, "Parameter estimation of chirp signals," IEEE Trans. Acoust., Speech, Signal Process., vol. 38, no. 12, pp. 2118-2126, Dec. 1990.

[8] B. Boashash, "Estimating and interpreting the instantaneous frequency of a signal," Proc. IEEE, vol. 80, no. 4, pp. 519-569, Apr. 1992.

[9] G.F. Boudreaux-Bartels and T.W. Parks, "Time-varying filtering and signal estimation techniques using Wigner distribution synthesis techniques," IEEE Trans. Acoust., Speech, Signal Process., vol. 34, no. 3, pp. 442-451, Jun. 1986

[10] S. Kay and G. F. Boudreaux-Bartels, On the optimality of the Wigner distribution for detecting a chirp signal, in Proc. IEEE ICASSP, Mar. 1985.

[11] L. Zheng and D. Shi, "Maximum amplitude method for estimating the compact fractional Fourier domain," IEEE Signal Proc. Lett., vol. 17, no. 3, Mar. 2010.

[12] C. Capus and K. Brown, "Fractional Fourier transform of the Gaussian and fractional domain signal support," IEE Proc. Vis. Image. Signal Process., vol. 150, no. 2, pp. 99-106, Apr. 2003.

[13] C. Capus, Y. Rzhanov, and L. Linett, "The analysis of multiple linear chirp signals," Proc. IEE Symposium on Time-Scale and TimeFrequency Analysis and Applications, pp. 4/1-4/7, London, Feb. 2000.

[14] D.E. Ferguson, "Fibonaccian searching," Commun. ACM, vol. 3, no. 12, Dec. 1960.

[15] L.B. Almeida, "The fractional Fourier transform and time frequency representations," IEEE Trans. Signal Process., vol. 42, no. 11, pp. 30843091, Nov. 1994.

[16] C. Candan, M.A. Kutay, and H.M. Ozaktas, "The discrete fractional Fourier transform," IEEE Trans. Signal Process., vol. 48, no. 5, pp. 13291337, May 2000.

\section{BIOGRAPHY}

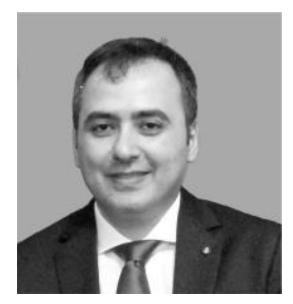

AHMET SERBES received the B.S. degree from Istanbul Technical University, Istanbul, Turkey in 2002, the M.S. and Ph.D. degrees from Yildiz Technical university in 2004 and 2011, respectively all in electronics and communications engineering.

$\mathrm{He}$ is currently with the department of Electonics and Communications Eng. Dept., Yildiz Technical University, where he is an Assistant Professor. His research interests include statistical signal processing, estimation and detection theory, communications, linear transformations, time-frequency signal processing, and fractional Fourier transform. 